\title{
Accumulation of small murine minor satellite transcripts leads to impaired centromeric architecture and function
}

\begin{abstract}
Haniaa Bouzinba-Segard, Adeline Guais, and Claire Francastel* F-75014 Paris, France
\end{abstract}

Département d'Hématologie, Institut Cochin, F-75014 Paris, France; Institut National de la Santé et de la Recherche Médicale, U567, F-75014 Paris, France; Centre National de la Recherche Scientifique, UMR 8104, F-75014 Paris, France; and Faculté de Médecine René Descartes, UM 3, Université Paris 5,

Edited by Mark T. Groudine, Fred Hutchinson Cancer Research Center, Seattle, WA, and approved May 2, 2006 (received for review September 13, 2005) localization at centromeric regions (17).
Other mechanisms may include direct binding to DNA or interaction with heterochromatin proteins. In addition, an RNA component was shown to be an integral component of human pericentromeric heterochromatin (16), and necessary for HP1

The centromere core is often viewed as a transcriptionally inactive domain of the chromosome. However, recent data suggest that transcription across centromeric repeats may contribute to centromere formation $(18-22)$ and indicate that genes within centromeres can be transcribed $(23,24)$. Transcription and/or remodeling of the nucleosomes at the centromeres may also be important for the deposition of CENP-A $(21,22)$. Alternatively, transcription across tandem repeats would favor the generation of self-templating dsRNA, further processed by the interference machinery (25). The demonstration that the RNA interference machinery is required for centromeric silencing suggested a role for short interfering (si)RNAs in mediating assembly of centromeric heterochromatin (26-29).

We report here that transcription of murine centromeric repeats, analyzed by Northern blot, produces small transcripts, $\approx 120$-nt long, which accumulate upon stress conditions and differentiation and localize on chromocenters. Stress-induced or forced accumulation of these 120-nt transcripts leads to chromosome missegregation, loss of sister-chromatid cohesion, and aneuploidy. We show that alterations in the epigenetic marks and in the localization of proteins associated with centromeric and pericentromeric regions correlate with the impaired centromeric function. These data suggest a role for small centromeric transcripts in the epigenetic regulation of murine centromere function and architecture.

\section{Results}

Minor Satellite Transcripts Accumulate as Small RNAs in Murine Cells. Murine minor satellites contain a 120-bp unit organized into uninterrupted blocks of tandem arrays of $\approx 2,500$ copies per chromosome. We analyzed the kinetics of transcription across murine centromeric repeats in murine erythroleukemic (MEL) cells, a murine model system widely used to study events associated with cellular differentiation/tissue-specific gene expression. Because these cells are immortalized leukemic cells, we also analyzed primary stromal cells (MS5) and a nonimmortalized myoblast cell line (C2C12). Given the repetitive nature of this region, PCR techniques cannot be reliably quantitative or give information about the size of the transcripts. We therefore

Conflict of interest statement: No conflicts declared.

This paper was submitted directly (Track II) to the PNAS office.

Abbreviations: MEL, murine erythroleukemic; siRNA, short interfering RNA

*To whom correspondence should be addressed at: Département d'Hématologie, Maternité Port Royal, Institut Cochin, 123, Boulevard Port-Royal, 75014 Paris, France. E-mail: francastel@cochin.inserm.fr.

() 2006 by The National Academy of Sciences of the USA 
A

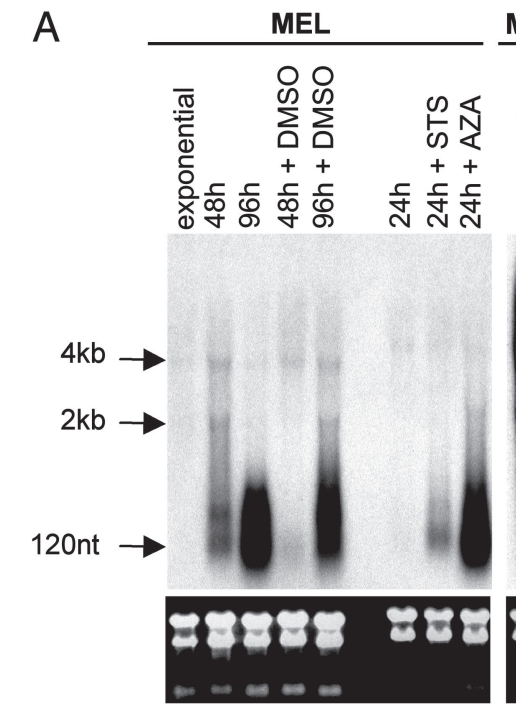

MS5

$\mathrm{C2C} 12$

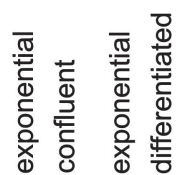

B

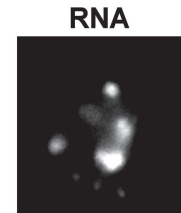

DAPI
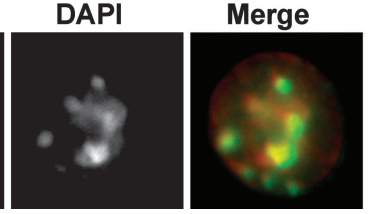

Fig. 1. The major detectable form of murine satellite repeats transcripts is 120-nt long. (A) Total RNA extracts from murine cell lines or primary cells were analyzed by Northern blot to determine the size and relative abundance of transcripts from centromeric minor satellite repeats in exponentially growing, confluent, or differentiated cells. The minor satellite repeat unit fragment, in the sense orientation, was used as a probe. The profile of ribosomal 28S, 18S, and $5 \mathrm{~S}$, stained with ethidium bromide, is shown below each blot to confirm equal loading of the lanes. Cell lines analyzed were MEL in exponentially growing phase or after 48 and $96 \mathrm{~h}$ in culture, with or without the inducer of differentiation (DMSO), or MEL cells treated for $24 \mathrm{~h}$ with the inhibitor of DNA methylation 5-azacytidine (AZA) or the inducer of apoptosis staurosporine (STS) (Left); murine primary stromal cells, exponentially growing or confluent myoblast cells (Center, MS5), and exponentially growing or differentiated myoblast cells (Right, C2C12). (B) RNA-FISH analysis on confluent MEL cells, using a biotinylated probe identical to the one used in Northern blots. (Left) Centromeric transcripts. (Center) DAPI. (Right) Merge of both images, with RNA in green and DAPI in red.

performed Northern blot analysis on total RNA isolated from the above-cited murine cells, in different growth and differentiation conditions, using the minor satellite 120-bp unit (6) in both orientations as a probe. We showed that minor satellites were indeed transcribed, as previously reported for other cell types $(18,19)$, as two long, yet barely detectable, 2 - and 4-kb precursor transcripts (Fig. 1A). Surprisingly, centromeric transcripts appeared to accumulate with time in culture, as smaller RNAs. In confluent MEL (96 h) and MS5 cells, the size of the major detectable type of centromeric transcripts was $\approx 120 \mathrm{nt}$. We checked that these RNAs were sensitive to RNase treatment but resistant to digestion with either DNases or RNaseH, to rule out the possibility that the observed small fragments may come from a DNA contamination or from a DNA fragmentation associated with the accumulation of apoptotic cells in the culture (see Fig. 4, which is published as supporting information on the PNAS web site). Thus, transcription of minor satellite DNA seemed to be temporally regulated because the smaller RNAs were undetectable in exponentially growing cells, appeared at day two of culture, and accumulated further as cells reached the growth plateau and became apoptotic. However, run-off experiments comparing exponentially growing and confluent MEL cells showed that small transcript accumulation did not result from an increased rate of transcription through satellite repeats (data not shown) and may result from an increased stability of these transcripts. Alternatively, their accumulation may also result from an increased rate of cleavage of the longer transcripts when cells reach the growth plateau.

The simultaneous presence of transcripts with sense and antisense sequences (29) (and see Fig. 5, which is published as supporting information on the PNAS web site) is expected to activate the RNA interference machinery and produce siRNAs (25). However, we were unable to detect significant levels of centromeric RNAs in the size range of 22-30 nt on polyacrylamide gels (see Fig. 6B, which is published as supporting information on the PNAS web site).

Minor Satellite Transcripts Accumulate Under Stress or Differentiation Conditions. We previously reported changes in the composition of centromeric heterochromatin compartments as MEL cells were induced to differentiate upon DMSO exposure (30). Here, we wanted to address whether changes in transcription through satellite repeats could be related to these changes. Northern blot analyses showed that centromeric transcripts accumulated as small RNAs during both DMSO-induced erythroid differentiation of MEL cells and myogenic differentiation of $\mathrm{C} 2 \mathrm{C} 12$ cells (Figs. 1A Right and 6). We also showed that the accumulation of these transcripts was induced by a 24-h treatment with 5-azacytidine, an inhibitor of DNA methylation, or by staurosporine, a known inducer of apoptosis (Fig. $1 A$ ). This result is in agreement with a report showing that age-related accumulation of DNA damage or demethylation of mouse satellites might facilitate transcription of these sequences (18).

Characterization of the subcellular localization of these transcripts by RNA-FISH, using a fluorescent probe identical to the one used on Northern blots, showed that spatial distribution of centromeric transcripts overlapped the bright DAPI foci known as chromocenters, i.e., clusters of several centromeres (Fig. 1B). It may be argued that the centromeric probe used may also hybridize with arrays of centromeric DNA repeats with similar sequences. If that were the case, we would detect strong signals in the majority of the cells. However, strong signal was detectable in a subset of cells and remained almost undetectable in the rest of the population. Moreover, it was shown that minor satellite repeats are detected at the periphery of DAPI-dense staining, whereas major satellites colocalize with these large spots (4). It appears then that centromeric transcripts accumulate on regions wider than the sites from which they are transcribed. The apparent association of centromeric transcripts with chromocenters, together with reports suggesting that an RNA component is important for heterochromatin assembly at centromeric regions $(16,17)$, prompted us to assess the contribution of small centromeric RNAs to centromere assembly and/or function.

Enforced Accumulation of 120-nt Centromeric Transcripts Impairs Centromeric Function. The bulk of centromeric transcripts being between 120 and $500 \mathrm{nt}$, we decided to explore the function of small 120-nt satellite transcripts. Because accumulation of centromeric transcripts was not homogeneous in the MEL cell population, we decided to use a transgene-based approach to enrich the population in cells expressing small centromeric transcripts, independently of the growth and differentiation conditions. MEL cells were transiently cotransfected with an expressing vector driving transcription of one minor satellite 120-bp repeat unit, together with a GFP-expressing vector to allow sorting of the cells on the basis of their GFP expression level into homogeneous populations of non- $\left(\mathrm{GFP}^{-}\right)$, low $\left(\mathrm{GFP}^{+}\right)$and high $\left(\mathrm{GFP}^{+++}\right)$expressing cells. We then checked that expression of small centromeric transcripts was proportional 
A
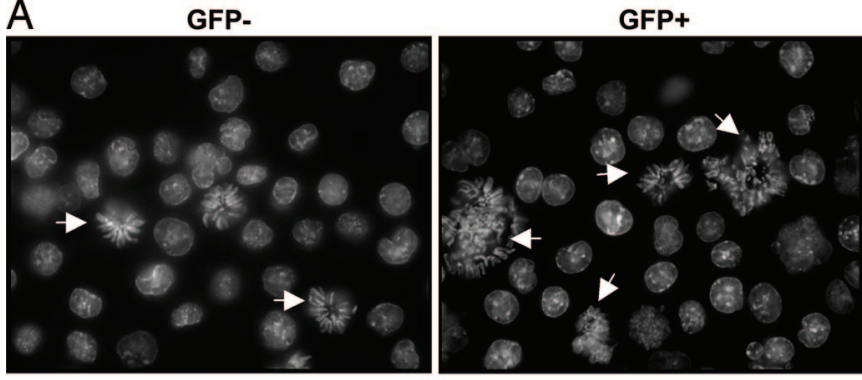

B
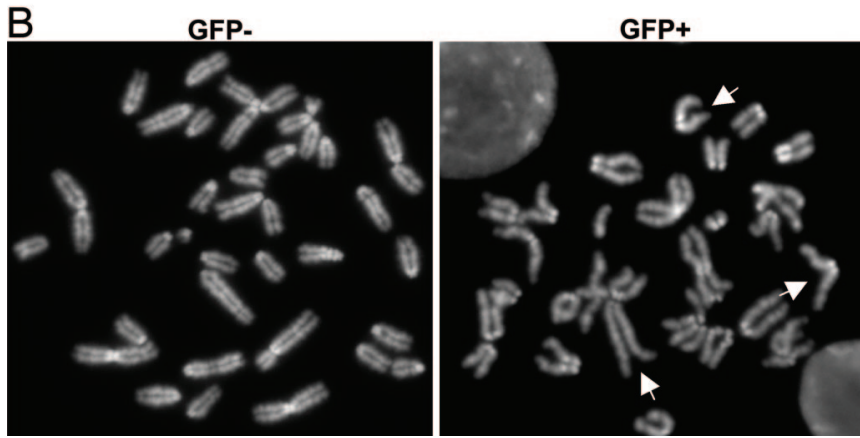

Fig. 2. Ectopic expression of satellite transcripts leads to impaired mitosis and lack of sister-chromatid cohesion. $(A)$ Examples of interphase and mitotic nuclei from pSAT-transfected cells after cell sorting. Cells were fixed and nuclei stained with DAPI. The images show normal mitotic figures in $\mathrm{GFP}^{-}$cells (Left, arrows) and accumulation of mitosis with misaligned chromosomes in pSAT/GFP ${ }^{+}$-transfected cells (Right, arrows). (B) Examples of DAPI-stained chromosomes from metaphase spreads. (Left) GFP ${ }^{-}$showing normal chromosome shape and chromatid cohesion. (Right) $\mathrm{pSAT} / \mathrm{GFP}^{+}$-transfected MEL showing impaired sister-chromatid cohesion and centromere cleavage (arrows).

to that of GFP (see Fig. 7, which is published as supporting information on the PNAS web site).

Visual analysis of the phenotype caused by accumulation of centromeric transcripts on fixed and DAPI-stained cells showed an increase in the number of total prometaphases and metaphases in the culture, the almost complete absence of anaphases, and an accumulation of micronuclei (Fig. $2 A$ ). Moreover, these metaphases were disorganized, showing misaligned and abnormal numbers of chromosomes, characteristic of a failure to undergo complete mitosis. The percentage of impaired mitotic figures counted in $\mathrm{GFP}^{-}, \mathrm{GFP}^{+}$and $\mathrm{GFP}^{+++}$was $10 \%$, $62 \%$, and $90 \%$ respectively, indicating that there was a good correlation between the accumulation of impaired mitotic figures and the amount of centromeric transcripts detected in transfected cells. We then analyzed mitotic figures more closely on metaphase spreads from cells expressing centromeric transcripts or not and observed important morphological changes of the chromosomes. As shown in Fig. $2 B$, chromosomes from centromeric RNA-expressing cells exhibited altered shape and condensation and a striking separation of sister chromatids compared with normal metaphasic chromosomes. Immunofluorescence experiments with an anti- $\beta$-tubulin antibody showed that the spindle could form, although most of the mitotic arrested cells had multiple spindles (Fig. $7 B$ ). In addition, the inner-kinetochore assembly pathway did not seem to be affected because localization of constitutive kinetochore proteins, CENP-B and CENP-C (Fig. 7B), was not altered in cells expressing ectopic centromeric transcripts. Together, our data show a defect in chromosome segregation and are consistent with an impaired centromere function associated with accumulation of small centromeric transcripts.

Enforced Accumulation of 120-nt Centromeric Transcripts Impairs Centromeric Architecture. To further characterize the possible cause of centromeric dysfunction, we labeled proteins known to play a role in either attachment of centromeres to microtubules or in sister-chromatid cohesion, using specific antibodies. Aurora-B, a member of the chromosomal passenger complex, which transiently associates with centromeres during prometaphase (31) (Fig. 3 Left), had lost its specific and restricted localization to centromeric regions upon ectopic expression of centromeric transcripts and seemed to be broadly distributed across the chromosomes (Fig. 3

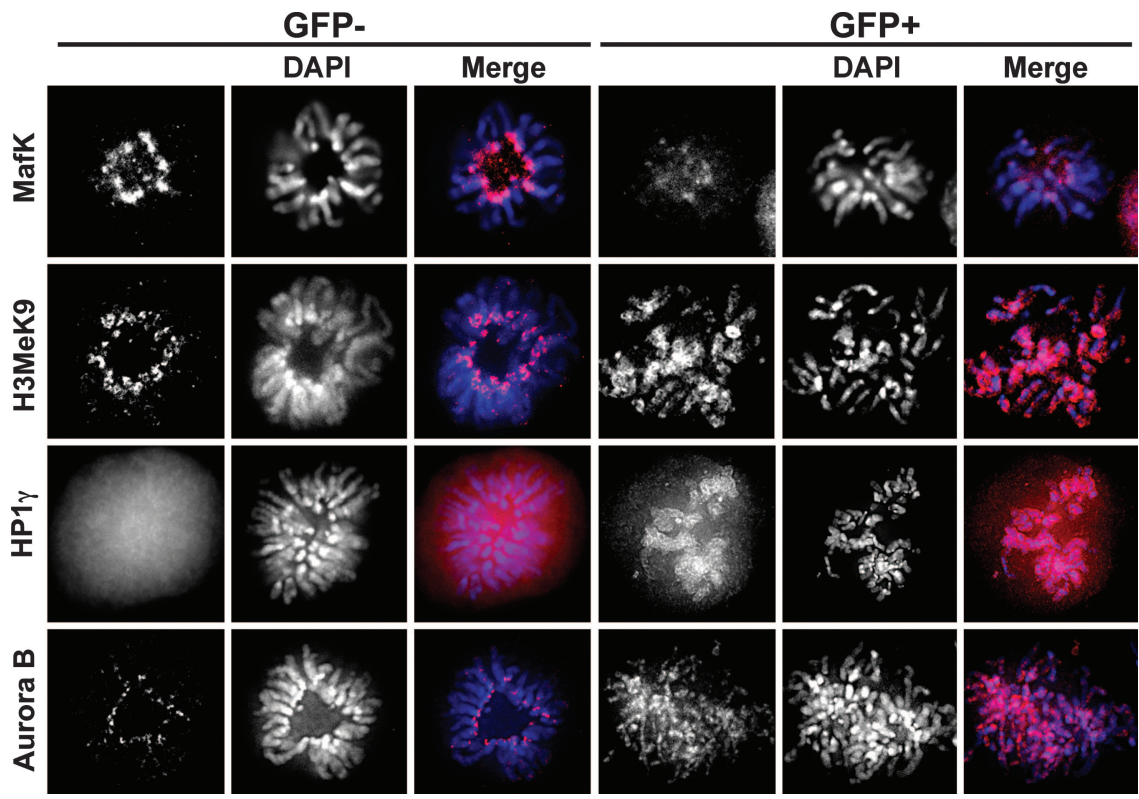

Fig. 3. Accumulation of centromeric transcripts leads to mislocalization of centromere-associated proteins. Immunofluorescence on pSAT-transfected MEL cells [GFP ${ }^{-}$cells (Left) and GFP' cells (Right)] using antibodies against (at left, from bottom to top) the chromosomal passenger protein Aurora-B, the centromereassociated protein HP1 $\gamma$, or the histone H3 trimethylated on K9 (H3MeK9) and MafK. Chromosomes are stained with DAPI (Center). Specific staining appears in red and chromosomes in blue in the merge lanes. 
Right). It was shown in yeast and in Drosophila that one important function for pericentromeric heterochromatin was its ability to recruit the cohesin complex required for sister-chromatid cohesion through interaction with $\operatorname{HP1}(32,33)$. Because we observed a lack of chromatid cohesion, we further analyzed heterochromatin composition at pericentromeric heterochromatin in our system and found that, whereas HP1 $\gamma$ was absent from control mitotic chromosomes, HP1 $\gamma$ was detected on chromosomes in mitotic figures deriving from cells expressing centromeric transcripts (Fig. 3). Consistent with this finding, histone $\mathrm{H} 3$ trimethylated on its Lys-9 (H3MeK9), involved in recruitment of HP1 proteins to heterochromatin (34) and associated with centromeric regions in control metaphases, also became mislocalized and showed broad localization on chromosome arms as cells expressed high levels of centromeric transcripts. Finally, we also analyzed the behavior of MafK, the previously studied factor required for MEL cell differentiation (30), and found that MafK also became absent from centromeric regions in cells accumulating centromeric transcripts.

Despite mitotic defects in cells expressing small centromeric transcripts, we observed an accumulation of cells presenting micronuclei and apoptotic cells, indicating that these cells eventually exit mitosis as polyploid or aneuploid cells. We thus investigated the heterochromatin architecture in interphase transfected cells and found that HP1 $\gamma$ staining did not show any striking difference to that of control cells, i.e., HP1 $\gamma$ still localized, although partially in some cells, to chromocenters (data not shown). Because this protein is very abundant and very stable in our system, delocalization of endogenous proteins might be difficult to observe during the course of a transient transfection. We thus decided to assess protein localization when ectopically expressed as a tagged protein, in interphase cells expressing small centromeric transcripts, to analyze the targeting of a newly synthesized protein. Detection of the exogenous protein, by using a specific antibody against the epitope tag, revealed that, in MEL cells, HP1 $\gamma$, as well as the previously described centromere-associated transcriptional repressor MafK are efficiently targeted to their normal location, i.e., chromocenters. However, in cells expressing high levels of small centromeric transcripts, both HP1 $\gamma$ and MafK were no longer targeted to centromeres (see Fig. 8, which is published as supporting information on the PNAS web site) and showed a rather diffuse pattern throughout the nucleus. This approach was also intended to study the behavior of the histone methyl transferase Suv39h1, which catalyses trimethylation of histone H3 on Lys-9 required for targeting of HP1 to heterochromatin, and against which we did not have good antibodies to detect the endogenous protein. We show that, whereas Suv39h1 localizes to chromocenters in control cells, as described in ref. 11, expression of small centromeric transcripts led to His mistargeting away from centromeric regions.

\section{Discussion}

Murine centromeric regions are known to be transcribed (18, $19)$, and recent data suggest that transcription in the satellite repeats may contribute to centromere assembly (20-22). Our characterization of transcription across murine minor satellite repeats shows that, in addition to large transcripts, murine centromeres produce smaller transcripts, ranging from 100- to 500-nt long. These small transcripts, undetectable in exponentially growing cells, accumulate upon stress and differentiation and colocalize with regions of dense DAPI staining. Stressinduced or forced accumulation of these 120-nt transcripts leads to altered epigenetic markers, impaired centromeric function, and further death of the cells. Our data suggest that the observed accumulation of small minor satellite transcripts is responsible for the centromere dysfunction by tethering centromereassociated proteins required for centromere architecture and function and raise the interesting possibility that these transcripts may serve as an additional mechanism for transcriptionregulated control of centromere assembly and function.

We identified small RNA molecules, in the range of 100-500 nt, recognized on Northern blots by a probe specific for minor satellite repeats in both orientations. Whether these RNAs originate from cleavage of a longer transcript or from multiple initiation sites is not known. Transcription across tandem repeats may favor generation of dsRNA that would be further cleaved as smaller RNAs by type III RNases. The exact arrangement of tandem repeats in satellite DNA is not clear; however, the existence in the EST database of transcripts containing the basic repeat in both orientations confirms that formation of dsRNA can originate from the same RNA molecule. Centromeric transcripts originating from both strands (ref. 29 and this study) could also favor formation of dsRNA. Another possibility is that folding of long multimeric precursor RNAs may create ribozyme-like structures with self-cleavage activity, the product of which is a monomer. Indeed, the size of the small RNAs, i.e., multiples of $120 \mathrm{nt}$, first determined by Northern blot is striking. RT-PCR analysis, using various combinations of primers along the repeat unit, showed further that these RNAs have the same length as the minor satellite repeat unit (120 nt) and that they may, indeed, represent monomers of the satellite repeats (data not shown). In addition, accumulation of small centromeric transcripts did not seem to be accompanied by a transcriptional activation in these regions. Taken together, our data suggest that small minor satellite transcripts may originate from the cleavage of longer species transcribed through contiguous repeats, although the nature and mechanisms of production of these small transcripts remain to be established.

We report the accumulation of small centromeric transcripts in stress conditions, i.e., lack of nutriments, DNA demethylation, or induction of apoptosis. Other examples exist that show increased transcription of satellite III repeats or perturbed heterochromatin structures at pericentromeric regions in response to heat shock (35). We also showed that centromeric transcripts accumulate during differentiation of two model systems, erythroid and myogenic cells, consistent with recent experiments showing that retinoic acid-induced differentiation of ES cells or terminal muscle differentiation also leads to increased transcript levels of satellite repeats $(29,36)$. We had previously reported the relocalization of the transcriptional repressor NF-E2/MafK away from centromeric compartments after induction of MEL cells, concomitant with relocalization and transcriptional activation of its target gene. Here, we show that ectopic accumulation of small centromeric transcripts leads to MafK relocalization away from centromeric regions, although it is not sufficient to promote differentiation of these cells (data not shown). Together, these data suggest that accumulation of noncoding centromeric transcripts maybe associated with changes in the nuclear organization of heterochromatin compartments that accompanies cellular differentiation. It appears, then, that satellite transcript-mediated alterations in the higherorder structure of heterochromatin regions could also induce the transcriptional activation of silent portions of the genome. Consistent with this idea, erythroid differentiation of MEL cells resulted in changes in the constitutive heterochromatin compartment and further activation of the erythroid program (30) in association with increased levels of centromeric transcripts (this study).

The simultaneous presence of transcripts with sense and antisense sequences is expected to activate the RNA interference machinery and produce siRNAs (25). However, we were unable to detect significant levels of centromeric RNAs in the size range of 22-30 nt on polyacrylamide gels. Whether these short RNAs are unstable or undetectable in our experimental conditions is not known. However, our Northern blot analysis showed that larger 
transcripts can be readily detected and that the most abundant minor satellite transcripts in our system are in the size range of 100-500 nt. As shown in plants, multiple forms for centromeric transcripts exist $(22,37)$, but, in the systems we studied, only the small 120-nt RNAs accumulate upon stress or differentiation, in correlation with subsequent centromere dysfunction. Because these small centromeric transcripts decorate DAPI-dense foci, constituted mainly of pericentromeric regions, it was tempting to address a potential role for these transcripts in centromere architecture and function. Forced expression of these small centromeric transcripts led to accumulation of mitotic figures containing misaligned chromosomes and defects in sister-chromatid cohesion generally associated with impaired centromere function. There is a clear specialization of satellite repeats during mitosis, minor satellites on which assembles the kinetochore being implicated in chromosome attachment to the spindle and major satellites associated with heterochromatin proteins recruiting cohesions. It is then conceivable that the phenotype we observed after accumulation of small centromeric transcripts, consistent with their broad localization over chromocenters, may result from mislocalization of both kinetochore- and heterochromatin-associated proteins, thereby impairing chromosome attachment to the spindle or sister-chromatid cohesion. We thus chose to analyze the localization on mitotic chromatin of two proteins representative of each group and for which a clear role in centromere function is known, namely the passenger protein Aurora-B $(38,39)$ and HP1 (40). We found that centromeric transcript accumulation resulted in dramatic changes in both $\mathrm{Au}-$ rora-B and HP1 $\gamma$ localization. Aurora-B had lost its specific and restricted localization on centromeric regions of mitotic chromosomes, with staining appearing on the chromosome arms, whereas we could not detect significant changes in phosphorylation of its mitotic substrate, the Ser-10 of histone H3 (not shown). HP1 $\gamma$, normally absent from mitotic chromosomes in MEL cells, remained associated with chromosomes. Consistent with the observed persistence of HP1 $\gamma$ on chromosomes, we found that, in cells expressing small centromeric transcripts, trimethylated histone $\mathrm{H} 3$ on Lys-9 (H3K9Me3) occupied a more broad distribution on chromosome arms and that targeting of the histone methyl transferase Suv39h1 to pericentromeric regions was impaired. It appears that accumulation of small centromeric transcripts leads to disruption of heterochromatin formation at centromeric regions and impairs recruitment of newly synthesized centromere-associated proteins.

There are now numerous examples suggesting that transcription and chromatin remodeling at centromeric repeats may be important for initiation and maintenance of kinetochore- and chromatin-associated proteins $(26,41,42)$. Since the discovery that short RNAs with centromeric sequences are part of the centromeric complex in yeast (43), numerous studies have subsequently envisioned a role for siRNAs in assembly of centromeric heterochromatin in mammalian systems and found that, in the absence of the RNA interference machinery, assembly of heterochromatin at centromeres is severely impaired (27, 28). A role as guiding molecules was proposed for these short RNAs to promote recruitment of histone methyl transferases and methylation of centromeric repeats required for their heterochromatin specification $(42,44,45)$. There are several other means, possibly implicating larger RNAs, by which transcription and/or centromeric RNAs could participate in specification of the chromatin structure at centromeres. Active transcription across centromeric repeats may affect the nucleosomal composition at centromeres and favor replacement of histone $\mathrm{H} 3$ by its variant CENP-A, the hallmark of functional centromeres (20). Another example in maize shows that centromeric transcripts coprecipitate with the histone H3 variant CenpH3 (CENP-A) $(21,22)$. Interestingly, expression of Xist RNA is sufficient to promote incorporation of macroH2A1, a histone variant found at the inactive X chromosome (46). Noncoding RNAs may then contribute to histone variant deposition at specific chromosomal regions, associated with early specification of these regions. This deposition could be achieved through direct binding of histone variants to these noncoding RNAs, although this remains to be demonstrated. Noncoding RNAs are also integral components and implicated in the stabilization of several large-scale chromatin protein complexes. For instance, RNA is a known component of sex chromosome dosage compensation complexes in mammals and Drosophila (47), of the yeast telomerase complex (48), the human pericentromeric heterochromatin (16), or more recently, the nuclear stress bodies containing RNA processing factors $(49,50)$. By analogy, noncoding centromeric RNAs may nucleate or serve as a scaffold for chromatin remodelingcontaining complexes at the centromere. The identification of proteins interacting with this type of RNAs remains to be done and promises to reveal interesting mechanisms in epigenetic control of centromere architecture and function.

In essence, and as recently demonstrated in yeast or in mice $(51,52)$, it appears that multiple pathways in the control of heterochromatin assembly and centromere specification in mammals exist. From our localization of small minor satellite transcripts on chromocenters, it is tempting to speculate that these centromeric RNAs belong to a nucleoprotein complex located at centromeric regions. It is then possible that accumulation of centromeric transcripts, in stress or differentiation conditions (our study and refs. 29 and 36) or after reduced levels of enzymes of the interference machinery $(27,28,52)$, could titrate centromere-associated proteins leading to their mislocalization away from their default localization site (our study). Although it cannot be excluded that accumulation of large centromeric RNAs in stress or differentiation conditions could simply interfere with production of siRNAs, our study provides evidence that larger RNAs can be responsible for the observed centromere dysfunction, aneuploidy, and further death of the cells. However, whether these transcripts are required for normal centromere function and participate in an epigenetic structure at the centromere remains to be seen.

\section{Methods}

Cell Culture and Transfection. MEL were grown and induced to differentiate as described (53). Murine C2C12 myoblasts and the stromal murine MS5 cells were grown in DMEM supplemented with $10 \%$ FCS. Differentiation of $\mathrm{C} 2 \mathrm{C} 12$ cells was induced by serum starvation. Where indicated, exponentially growing cells were treated with $1 \mu \mathrm{M} 5$-azacytidine (Sigma), or $0.2 \mu \mathrm{M}$ staurosporine (Sigma) during $24 \mathrm{~h}$. Transfection of MEL cells was performed by electroporation $(250 \mathrm{~V}, 960 \mu \mathrm{F}$ with a Gene Pulser, Bio-Rad) and MS5 with Fugene 6 (Roche) according to the manufacturer's instructions. Plamids used for transfections are described in Supporting Methods, which is published as supporting information on the PNAS web site.

RNA-FISH. Cells were washed in PBS, deposited on a Superfrost Plus microscope slide (Menzel-Glaser, Braunschweig, Germany) at 50,000 cells per $100 \mu \mathrm{l}$ at room temperature (RT) for $10 \mathrm{~min}$, fixed for $10 \mathrm{~min}$ at RT with $4 \%$ paraformaldehyde (Electron Microscopy Sciences, Hatfield, PA), permeabilized for 5 min with $0.001 \%$ pepsine (Sigma) in $10 \mathrm{mM} \mathrm{HCl}$, and postfixed with $1 \%$ paraformaldehyde. An additional RNaseA treatment (Roche, $100 \mu \mathrm{g} / \mathrm{ml}$ for $30 \mathrm{~min}$ ) was added on control slides. Slides were hybridized overnight at $37^{\circ} \mathrm{C}$ with $10 \mathrm{ng}$ of the biotinylated minor satellite oligonucleotide probe (Supporting Methods), 20 units of RNaseOUT (Invitrogen), $2 \mu \mathrm{g}$ of salmon sperm DNA (Sigma), $2 \times \mathrm{SSC}(0.3 \mathrm{M} \mathrm{NaCl}$ and $30 \mathrm{mM}$ sodium citrate), $1 \mathrm{mM}$ EDTA, and $5 \times$ Denhardt's solution. Slides were washed three times for $10 \mathrm{~min}$ in $2 \times \mathrm{SSC}$ at $37^{\circ} \mathrm{C}$, revealed by using Alexa Fluor 594-conjugated streptavidin (1:1,000; Molecular Probes), and mounted with Vectashield/DAPI (Vector Laboratories). 
Immunofluorescence. The immunostainings, in conditions that preserve nuclear architecture, were performed as described (30) by using the antibodies described in Supporting Methods. Image acquisition was performed on an Axioplan 2 fluorescence microscope (Zeiss) and analyzed with the METAMORPH 5.6 software (Roper Scientific, Trenton, NJ).

Metaphase Spread. Cells were swollen in $75 \mathrm{mM} \mathrm{KCl}$ for $25 \mathrm{~min}$ and fixed in three changes of $10 \mathrm{~min}$ in methanol/acetic acid (3:1). Cells were then resuspended at $\approx 1 \times 10^{6}$ cells per $\mathrm{ml}$, and $100 \mu \mathrm{l}$ were projected on Starfrost slides (Menzel-Glaser) and DNA stained with DAPI.

1. Choo, K. H. (1997) The Centromere (Oxford Univ. Press, New York).

2. Henikoff, S., Ahmad, K., Platero, J. S. \& van Steensel, B. (2000) Proc. Natl. Acad. Sci. USA 97, 716-721.

3. Vafa, O. \& Sullivan, K. F. (1997) Curr. Biol. 7, 897-900.

4. Guenatri, M., Bailly, D., Maison, C. \& Almouzni, G. (2004) J. Cell Biol. 166, 493-505.

5. Sullivan, B. A. \& Karpen, G. H. (2004) Nat. Struct. Mol. Biol. 11, 1076-1083.

6. Wong, A. K. \& Rattner, J. B. (1988) Nucleic Acids Res. 16, 11645-11661.

7. Joseph, A., Mitchell, A. R. \& Miller, O. J. (1989) Exp. Cell Res. 183, 494-500.

8. Vigneron, S., Prieto, S., Bernis, C., Labbe, J. C., Castro, A. \& Lorca, T. (2004) Mol. Biol. Cell 15, 4584-4596.

9. Craig, J. M., Earle, E., Canham, P., Wong, L. H., Anderson, M. \& Choo, K. H. (2003) Hum. Mol. Genet. 12, 3109-3121.

10. Bachman, K. E., Rountree, M. R. \& Baylin, S. B. (2001) J. Biol. Chem. 276, 32282-32287.

11. Aagaard, L., Laible, G., Selenko, P., Schmid, M., Dorn, R., Schotta, G., Kuhfittig, S., Wolf, A., Lebersorger, A., Singh, P. B., et al. (1999) EMBO J. 18, 1923-1938.

12. Jorgensen, H. F. \& Bird, A. (2002) Ment. Retard. Dev. Disabil. Res. Rev. 8, 87-93.

13. Fuks, F., Hurd, P. J., Wolf, D., Nan, X., Bird, A. P. \& Kouzarides, T. (2003) J. Biol. Chem. 278, 4035-4040.

14. Bannister, A. J., Zegerman, P., Partridge, J. F., Miska, E. A., Thomas, J. O., Allshire, R. C. \& Kouzarides, T. (2001) Nature 410, 120-124.

15. Lachner, M., O'Carroll, D., Rea, S., Mechtler, K. \& Jenuwein, T. (2001) Nature 410, 116-120.

16. Maison, C., Bailly, D., Peters, A. H., Quivy, J. P., Roche, D., Taddei, A., Lachner, M., Jenuwein, T. \& Almouzni, G. (2002) Nat. Genet. 30, 329-334.

17. Muchardt, C., Guilleme, M., Seeler, J. S., Trouche, D., Dejean, A. \& Yaniv, M. (2002) EMBO Rep. 3, 975-981.

18. Gaubatz, J. W. \& Cutler, R. G. (1990) J. Biol. Chem. 265, 17753-17758.

19. Rudert, F., Bronner, S., Garnier, J. M. \& Dolle, P. (1995) Mamm. Genome 6, 76-83.

20. Chen, E. S., Saitoh, S., Yanagida, M. \& Takahashi, K. (2003) Mol. Cell 11, 175-187.

21. Nakano, M., Okamoto, Y., Ohzeki, J. \& Masumoto, H. (2003) J. Cell Sci. 116, 4021-4034.

22. Topp, C. N., Zhong, C. X. \& Dawe, R. K. (2004) Proc. Natl. Acad. Sci. USA 101, 15986-15991.

23. Ekwall, K., Olsson, T., Turner, B. M., Cranston, G. \& Allshire, R. C. (1997) Cell 91, 1021-1032.

24. Wong, L. H., Saffery, R., Anderson, M. A., Earle, E., Quach, J. M., Stafford, A. J., Fowler, K. J. \& Choo, K. H. (2005) J. Biol. Chem. 280, 3954-3962.

25. Hutvagner, G. \& Zamore, P. D. (2002) Curr. Opin. Genet. Dev. 12, 225-232.

26. Volpe, T., Schramke, V., Hamilton, G. L., White, S. A., Teng, G., Martienssen, R. A. \& Allshire, R. C. (2003) Chromosome Res. 11, 137-146.
We thank Paul-Henri Roméo for constant support, Mark Groudine and Steven Kosak for critical comments on the data and the manuscript, Thierry Grange and Saadi Khochbin for discussion, anonymous reviewers for insightful comments, Régine Losson (Institut de Génétique et de Biologie Moléculaire et Cellulaire, Illkirch, France) and Thomas Jenuwein (Institute of Molecular Pathology, Vienna) for expression vectors, and the people from the Cytometry Facility at the Institut Cochin. This work was supported by the Institut National de la Santé et de la Recherche Médicale "Avenir" program and by grants from the Association pour la Recherche contre le Cancer, the Ligue Nationale Contre le Cancer, the Cent pour Sang la Vie, and the Fondation de France (to C.F.) and a postdoctoral fellowship from the Ligue Nationale Contre le Cancer (to A.G.).

27. Fukagawa, T., Nogami, M., Yoshikawa, M., Ikeno, M., Okazaki, T., Takami, Y., Nakayama, T. \& Oshimura, M. (2004) Nat. Cell Biol. 6, 784-791.

28. Kanellopoulou, C., Muljo, S. A., Kung, A. L., Ganesan, S., Drapkin, R., Jenuwein, T., Livingston, D. M. \& Rajewsky, K. (2005) Genes Dev. 19, 489-501.

29. Martens, J. H., O’Sullivan, R. J., Braunschweig, U., Opravil, S., Radolf, M., Steinlein, P. \& Jenuwein, T. (2005) EMBO J. 24, 800-812.

30. Francastel, C., Magis, W. \& Groudine, M. (2001) Proc. Natl. Acad. Sci. USA 98, 12120-12125.

31. Vagnarelli, P. \& Earnshaw, W. C. (2004) Chromosoma 113, 211-222.

32. Bernard, P., Maure, J. F., Partridge, J. F., Genier, S., Javerzat, J. P. \& Allshire, R. C. (2001) Science 294, 2539-2542.

33. Nonaka, N., Kitajima, T., Yokobayashi, S., Xiao, G., Yamamoto, M., Grewal, S. I. \& Watanabe, Y. (2002) Nat. Cell Biol. 4, 89-93.

34. Nakayama, J., Rice, J. C., Strahl, B. D., Allis, C. D. \& Grewal, S. I. (2001) Science 292, 110-113.

35. Rizzi, N., Denegri, M., Chiodi, I., Corioni, M., Valgardsdottir, R., Cobianchi, F., Riva, S. \& Biamonti, G. (2004) Mol. Biol. Cell 15, 543-551.

36. Terranova, R., Sauer, S., Merkenschlager, M. \& Fisher, A. G. (2005) Exp. Cell Res. 310, 344-356.

37. May, B. P., Lippman, Z. B., Fang, Y., Spector, D. L. \& Martienssen, R. A. (2005) PLoS Genet. 1, e79.

38. Murata-Hori, M. \& Wang, Y. L. (2002) Curr. Biol. 12, 894-899.

39. Ditchfield, C., Johnson, V. L., Tighe, A., Ellston, R., Haworth, C., Johnson, T., Mortlock, A., Keen, N. \& Taylor, S. S. (2003) J. Cell Biol. 161, 267-280.

40. Eissenberg, J. C. \& Elgin, S. C. (2000) Curr. Opin. Genet. Dev. 10, 204-210.

41. Almeida, R. \& Allshire, R. C. (2005) Trends Cell Biol. 15, 251-258.

42. Bernstein, E. \& Allis, C. D. (2005) Genes Dev. 19, 1635-1655.

43. Reinhart, B. J. \& Bartel, D. P. (2002) Science 297, 1831

44. Jenuwein, T. (2002) Science 297, 2215-2218.

45. Allshire, R. (2002) Science 297, 1818-1819.

46. Rasmussen, T. P., Mastrangelo, M. A., Eden, A., Pehrson, J. R. \& Jaenisch, R. (2000) J. Cell Biol. 150, 1189-1198.

47. Wutz, A. (2003) BioEssays 25, 434-442.

48. Zappulla, D. C. \& Cech, T. R. (2004) Proc. Natl. Acad. Sci. USA 101, 10024-10029.

49. Metz, A., Soret, J., Vourc'h, C., Tazi, J. \& Jolly, C. (2004) J. Cell Sci. 117, 4551-4558.

50. Valgardsdottir, R., Chiodi, I., Giordano, M., Cobianchi, F., Riva, S. \& Biamonti, G. (2005) Mol. Biol. Cell 16, 2597-2604.

51. Jia, S., Noma, K. \& Grewal, S. I. (2004) Science 304, 1971-1976.

52. Murchison, E. P., Partridge, J. F., Tam, O. H., Cheloufi, S. \& Hannon, G. J. (2005) Proc. Natl. Acad. Sci. USA 102, 12135-12140.

53. Francastel, C., Augery-Bourget, Y., Prenant, M., Walters, M., Martin, D. I. \& Robert-Lezenes, J. (1997) Oncogene 14, 873-877. 